\title{
Schistosomiasis and Infertility: What Do We Know?
}

\author{
Ana Rita Ribeiro, ${ }^{1,2}$ Carla Luis, ${ }^{3}$ Ruben Fernandes, ${ }^{1,2}$ and Monica C. Botelho $2,4, \star$
}

There is increasing attention on the complex interactions occurring between schistosome parasites and their hosts. However, little is known about the occurrence, epidemiology, and mechanisms of schistosomiasis-associated infertility. In this article, we argue that an in-depth understanding of the interplay between parasites and the host endocrine system may significantly enhance current knowledge of infertility in infected individuals. We discuss the basic hormonal mechanisms that may lead to the discovery of entirely novel anthelmintic interventions against schistosomiasis.

\section{Schistosomiasis and Infertility}

Infertility is defined as the incapacity to become pregnant after 12 months of regular and unprotected intercourse. This is a major public health problem that affects about one in six couples (15-20\%) worldwide and $12-30 \%$ of couples in sub-Saharan Africa [1-3]. It is well recognized as a major sequela of genital tract infection; many women in Africa with this ailment (85-90\%) suffer from subsequent infertility, and the cause can be attributed to pelvic infections [4]. Numerous diseases, including febrile diseases, sexually transmitted infections (STDs), and various parasitic infections, such as microfilaria and schistosomiasis, can cause infertility [5].

When considering the diagnosis of infertility there are two main features of this disease: primary infertility, considered when the woman never conceived, and secondary infertility when she had had a previous labor [5]. Most articles addressing infertility-associated schistosomiasis describe only primary infertility, and we found only two reports in the literature describing secondary infertility: one from our group [5] and one from another group [6], both reporting a lack of association between secondary infertility and schistosomiasis.

\section{Epidemiology and Pathogenesis of Genital Manifestations of Schistosomiasis}

As stated, schistosomiasis affects millions of people worldwide. About 20 million have severe disease, and the risk of infection affects 600 million others, including travelers from developed countries (Box 1) [7-9]. Both sexes can develop genital tract pathologies, but the prevalence is significantly higher in women $[10,11]$. The pathogenesis of this disease in the genital tract is thought to be caused by ova deposition and ensuing inflammation causing mechanical blockage, scar tissue, and destruction of anatomical structures [12,13]. Infection of the genital tract is most commonly caused by the Schistosoma haematobium species. The eggs are laid by adult worms residing in vasculature and they migrate to the bladder, and it is in this organ that the main pathology occurs. However, S. haematobium eggs can be deposited along the genitourinary tract such as the cervix and vagina in women, and testes in men, due to the proximity of the genital venous plexus (see Glossary), which allows easy parasitic migration. [10,14-18].

Schistosomiasis is not essentially a thromboembolic disease (i.e., defined as embolic events within the vasculature), but its eggs may lead to occlusion of the terminal veins and impairment of the blood supply [19]. It is widely described as a granulomatous disease, with eggs becoming lodged in tissue which then leads to inflammatory reactions and fibrosis. It is believed that infertility could be related to this tissue scarring, ensuing inflammation, and granulomatous reactions, due to the release of proteolytic enzymes by the ova, causing mechanical blockage, scar tissue, and destruction of anatomic structures $[12,20,21]$. As mentioned, women's infected ovaries can become scarred and fibrous, and Fallopian tubes may show nodular lesions. Therefore, this fibrosis of the ovaries and/or tubal occlusion, caused by granulomas, is thought to lead to infertility [2224]. This fibrosis could be explained by the egg granuloma composition of eosinophils, mononuclear phagocytes, fibroblasts, lymphocytes, neutrophils, plasma cells, and mast cells. The systemic

${ }^{1}$ Ciências Químicas e das Biomoléculas, Escola Superior de Saúde, Instituto Politécnico do Porto, Porto, Portugal ${ }^{2}$ Instituto de Investigação e Inovação em Saúde da Universidade do Porto (i3S), Porto, Portugal

${ }^{3}$ Departamento de Biomedicina, Unidade de Bioquímica, Faculdade de Medicina, Universidade do Porto, Porto, Portugal ${ }^{4}$ Instituto Nacional de Saúde Dr Ricardo Jorge (INSA), Porto, Portugal

*Correspondence:

monicabotelho@hotmail.com, monica.botelho@insa.min-saude.pt 


\section{Box 1. Global Schistosomiasis Status}

The neglected tropical diseases (NTDs) are a group of chronic, disabling, and disfiguring conditions that occur most commonly in the setting of extreme poverty, especially among the rural poor and some disadvantaged urban populations [52]. One of these NTDs, schistosomiasis, is sometimes referred to as bilharzias, bilharziasis, or snail fever. Schistosomiasis was discovered by Theodore Bilharz, a German surgeon, and is considered a parasitic helminthic NTD. Like many tropical diseases, it is endemic in areas where poor living conditions and poverty are prevalent. Because these parasites are water-borne species, populations that primarily rely on agriculture and fishing for their livelihood are at the highest risk of contamination [32,40,53]. Schistosomiasis remains a major public health problem. It is endemic in 75 countries, and most cases of schistosomiasis occur in Africa, making this condition one of the most common NTDs in the region $[14,15,54]$.

The World Health Organization (WHO) estimates that schistosomiasis affects at least 220.8 million people worldwide. This disease can cause chronic anemia and inflammation associated with a severe disability burden among children, adolescents, and young adults and has an incidence of 200000 deaths yearly [55,56]. Trapped ova induce a chronic granulomatous response that causes local and systemic pathological effects ranging from anemia, growth stunting, impaired cognition, and decreased physical fitness, with largely unknown effects on endocrinological hormones and their receptors, to organ-specific effects such as severe hepatosplenism and portal fibrosis with portal hypertension $[16,32,56]$

immune response is triggered by cercariae, adult worms, and eggs, and their antigens are transported to lymphoid organs, where they are captured, processed, and presented to antigen-specific $T$ cells which leads to $T$ cell activation and lymphokine secretion. Thus, systemic activation of immune responses ensues, in this way, triggering of chronic fibrosis, tubal adhesions, and possibly infertility. Anything which leads to an inflammatory response, such as an infection in the pelvis, pelvic inflammatory disease, or even a ruptured appendix, can trigger adhesion formation. Adhesions, also called scar tissue, can block or distort the Fallopian tubes [22,23,25]. This could also explain infertility associated with Schistosoma mansoni, which characteristically lays its eggs on the mesenterium plexus, and is therefore not able to cause infertility by a direct or mechanical cause. In fact, our group has demonstrated that mice infected with S. mansoni have impaired reproduction in terms of diminishing gestational length, number of pups, and histopathological alterations in reproductive organs [26].

In men, the symptoms include epididymitis (an inflammation of the epididymis at the back of the testicle) which can simulate tuberculosis and associated funiculitis, indolence, and possible formation of a fistula, hematospermia, pain during urination, prostatitis, and other effects. In men, reproduction is affected by schistosomiasis by the predisposition to secondary infection of the prostate and seminal vesicles. Sometimes it directly affects the testicles. As this is a solid organ, the trapped eggs induce a granulomatous reaction which leads to fibrosis in the case of $S$. haematobium infection $[10,11]$ and metaplasia and necrosis in the case of $S$. mansoni infection [26].

Genital schistosomiasis is also linked to horizontal transmission of HIV and AIDS. The presence of eggs in the lower genital tract, which includes the cervix, vulva, and vagina, gives rise to a wide array of symptoms in affected women, and these lesions, if located in the vagina or vulva, might lead to hypertrophy, and can grow for months or years. Consequently, the capacity of the epithelium to act as a physical barrier to viral transmission is probably reduced, leading to the possibility of transmission and propagation of HIV. This happens because vaginal and cervical lesions tend to bleed easily, so that the HIV present in semen would have direct access to the blood circulation and, through ulcerative lesions, to the regional lymph nodes $[27,28]$.

Hybrid schistosomes are particularly worrying. Experimental studies have revealed that interspecific hybridization might enhance infectivity and virulence. Also, hybrids can have wider host spectrums, potentially expanding their epidemiologic consequences [29]. At present, there is no report addressing hybrids associated with infertility, but given the fact that these hybrids give rise to
Glossary

Angiogenesis: the formation and development of new blood vessels.

Catechol-estrogen: a steroidal estrogen that contains catechol (1,2-dihydroxybenzene) within its structure. The catechol estrogens are endogenous metabolites of estradiol.

Disability burden: a concept to describe the impact of a condition on the affected population, that is comprised of a disability (YLD) and a mortality (YLL) component. Estimates of disability burden are used to compare and rank the impact of different conditions. Dyspareunia: painful or difficult sexual intercourse, especially in women.

Dysuria: the experience or condition of experiencing pain while discharging urine.

Fistula: an abnormal connection or passageway between organs or vessels that normally do not connect.

Funiculitis: inflammation of a funiculus, especially of the spermatic cord.

Hematospermia: the presence of blood in the semen.

Hematuria: the presence of blood in the urine.

Mass spectrometry: an analytical technique by which chemical substances are identified by the sorting of ions in electric and magnetic fields according to their mass-to-charge ratios.

Mesenterium: a contiguous set of tissues that attaches the intestines to the posterior abdominal wall.

Metabolite: a chemical substance that is a product of metabolic action or that is involved in a metabolic process.

Neglected tropical diseases

(NTDs): a diverse group of 20 communicable diseases recognized by the World Health Organization (WHO) as being underfunded relative to other conditions of equal prevalence; NTDs affect predominantly the world's poorest populations, and many have a chronic course, are physically disabling, and/or are associated with stigmatization. Pampiniform plexus: a network of many small veins found in the human male spermatic cord.

Plexus: a branching network of the vessels or nerves. 
characteristically ectopic egg locations it might increase the association of S. mansoni infertility both in females and males.

\section{Mechanisms Underlying Female and Male Infertility-Associated Schistosomiasis Female}

Schistosomiasis hematobia remains a significant risk factor because several cases of infertility in schistosomiasis-endemic areas have been attributed to female genital schistosomiasis (FGS) $[30,31]$. FGS is defined as the existence of schistosome eggs in the female reproductive system. The eggs cause chronic inflammation in the surrounding genital tissue, comprised of granulomas, fibrosis, and angiogenesis. Together with this, girls and women can experience hematuria, dysuria, urinary frequency, and an increased risk of bladder cancer [12]. 'Classic' lesions of FGS, which consist of eggs and granulomas associated with increased angiogenesis and inflammation, are described as 'sandy patches'. Sandy patches usually bleed on contact and can cause genital itching and pain, stress incontinence, dyspareunia, and possibly infertility [14].

FGS can be associated with endometritis, and is likely to cause female infertility. Urogenital schistosomiasis, in individuals of reproductive age, remains highly prevalent and underdiagnosed [15,32]. Lesions in the upper genital tract, which includes the uterus, Fallopian tubes, and ovaries, are less discernible by routine clinical examination [27]. Fibrosis of the ovaries or tubal occlusion caused by granulomas, due to ovarian, tubal, and uterine schistosomiasis, are thought to lead to infertility (Table 1) $[27,28]$. S. mansoni is also likely to cause infertility in women, although there are very few reports in the literature. In fact there are only two reports describing an ectopic location of $S$. mansoni eggs in the endocervix [32] and in the endometrium and ovary [33], and another two reports describing hormonal imbalance in female mice infected with S. mansoni [34,35]. Hormonal disturbances may also explain female infertility and subfecundity associated with S. mansoni infection (Table 1) $[2,5,26]$.

\section{Male}

Male infertility due to schistosomiasis hematobia, unlike female infertility, is poorly reported and can develop through different mechanisms: (i) testicular, in which there is direct damage of testicular tissue by bilharzial inflammation and granuloma formation; (ii) post-testicular, due to obstruction of excurrent genital ductal system at any region between the rete testis and ejaculatory ducts, and/or affection of the accessory sex organs (prostate, seminal vesicles) by bilharzial inflammation and granuloma formation, resulting in severe oligozoospermia (partial obstruction), azoospermia (complete obstruction), or subfertile semen parameters [36-39].

Worms can reach the veins of the spermatic cord, epididymis, and testis through several routes: (i) connection between the superior mesenteric and right spermatic veins, or along those between the inferior mesenteric and left spermatic veins; (ii) connection between the deferential vein and pelvic veins; (iii) connection between the pampiniform plexus in the scrotum and pelvic veins that cross near the external inguinal ring; (iv) direct spread from the epididymis to the testis (possible but remote); (v) systemic arterial spread, as observed in cases of ectopic bilharzial lesions (possible but remote) [8].

The trapped Schistosoma eggs, in men, lead to occlusion of the spermatic venous plexus and subsequent granuloma formation that may result in testicular infarction; these trapped eggs may also lead to intense granulomatous epididymitis and inhibition of spermatogenesis, causing male infertility $[38,40]$. Seminal vesiculitis can cause hematospermia, painful ejaculation, burning micturition, and low backache (Table 1) [38-40]. Once the disease has progressed to the granuloma stage, the damage is irreversible [38]. Comparable with female S. mansoni infection, there are very few documented cases of S. mansoni infection causing infertility in men. We found two reports describing ectopic testicular S. mansoni eggs $[39,40]$ and two reports describing male sex hormone imbalance $[41,42]$. In male animals infected with S. mansoni, again, only two reports describe hormonal imbalance: the work of Lansoud-Soukate et al. [43] corroborated recently by our own work [35] (Table 1).
Quinones: oxidized derivatives of reactive aromatic compounds with electron-donating substituents such as catechols. Seminal vesiculitis: an uncommon entity characterized by inflammation of the seminal vesicles. It is most commonly infective in etiology and often associated with concurrent infection elsewhere in the male genital tract. 
Table 1. Effects of Schistosomiasis in Female and Male Reproductive Organs

\begin{tabular}{|c|c|c|c|c|}
\hline & \multirow[t]{2}{*}{ Organs affected } & \multirow[t]{2}{*}{ Effects } & \multicolumn{2}{|c|}{ Study model } \\
\hline & & & Schistosoma haematobium & Schistosoma mansoni \\
\hline \multirow[t]{5}{*}{ Female } & Ovaries & $\begin{array}{l}\text { Hypogonadism, retarded puberty } \\
\text { Infertility (primary/secondary) }\end{array}$ & Human $[5,11-13,19,27,28,42]$ & $\begin{array}{l}\text { Mouse }[26,35] \\
\text { Human }[33]\end{array}$ \\
\hline & Tubes & $\begin{array}{l}\text { Ectopic/tubal pregnancy } \\
\text { Tubal abortion } \\
\text { Hemoperitoneum }\end{array}$ & Human $[3,11,17,19,23,24,27,28,30,31,54]$ & Mouse [26] \\
\hline & Uterus & $\begin{array}{l}\text { Anemia due to chronic blood loss } \\
\text { Metaplasia } \\
\text { Miscarriage, preterm delivery }\end{array}$ & Human $[22,27,28]$ & $\begin{array}{l}\text { Human }[33] \\
\text { Mouse [34] }\end{array}$ \\
\hline & Cervix & $\begin{array}{l}\text { Anemia due to chronic blood loss } \\
\text { Carcinoma } \\
\text { Risk for sexually transmitted disease }\end{array}$ & Human $[7,11,18,23,24,27,28,37]$ & Human $[28,32]$ \\
\hline & Vagina/vulva & $\begin{array}{l}\text { Destruction of hymen and/or clitoris } \\
\text { Vesico-vaginal fistula } \\
\text { Risk for sexually transmitted disease }\end{array}$ & $\begin{array}{l}\text { Mouse [16] } \\
\text { Human }[18,23,24,27,28,37]\end{array}$ & \\
\hline \multirow[t]{3}{*}{ Male } & Testicles & $\begin{array}{l}\text { Hypogonadism, retarded puberty } \\
\text { Infertility (primary/secondary) }\end{array}$ & Human $[9,42]$ & $\begin{array}{l}\text { Human }[20,39-42] \\
\text { Mouse }[26,35,43]\end{array}$ \\
\hline & Epididymis & $\begin{array}{l}\text { Pain, funiculitis, fistulization, } \\
\text { hemospermia, azoospermia }\end{array}$ & Human $[9,38]$ & Human [39] \\
\hline & Prostate & Pain during urination & Human [10] & \\
\hline
\end{tabular}

\section{Estrogen Receptors (ERs) and Estrogen Metabolism in Schistosomiasis}

Estrogens exert several biological effects in humans, such as in the cardiovascular, musculoskeletal, immune, and central nervous systems, and they play an important role in the development and maintenance of normal sexual and reproductive function [44]. Estrogen signaling is primarily mediated through ERs, which incorporate the classical receptors $E R \alpha$ and $E R \beta$ and the newly found membrane receptor $G$ protein-coupled estrogen receptor 30 (GPER30) [45]. ER $\alpha$ is mainly expressed in the gonadal organs (uterus, ovary, prostate, testes, and breast), liver, and adipose tissue. ER $\beta$ is found mostly in the prostate, bladder, ovary, colon, adipose tissue, and immune system. These classical receptors have common physiological roles such as in the development and function of the ovaries, and estrogen-mediated cardioprotective actions $[45,46]$.

GPER30, a member of the G-protein-coupled receptor superfamily, has been recognized as a membrane estrogen receptor which can trigger rapid estrogen nongenomic signaling independent of ER $\alpha$ and ER $\beta$. GPER30 is expressed in many brain regions, including the hippocampus, cortex, hypothalamus, specific nuclei within the midbrain, and the Purkinje layer of the cerebellum, as well as the adrenal medulla, renal pelvis, and ovaries $[45,47]$. Many studies suggest that there are a number of pathways by which estrogens and their receptors regulate biological processes (Box 2).

While addressing schistosomiasis-induced hypogonadism in male patients infected with S. haematobium and S. mansoni, Botelho et al. [42] observed a noteworthy elevation in serum levels of estradiol (E2), whereas those of luteinizing hormone (LH) and follicle stimulating hormone (FSH) remained normal, and hypothesized that the excess E2 could be external to the host. In fact, we found by mass spectrometry that the molecule responsible for the effect was an $\mathrm{S}$. haematobium-derived E2-like molecule that is an antagonist of E2 and thus repressed the transcriptional activity of the ER. 


\section{Box 2. Pathways by Which Estrogens and Their Receptors Regulate Biological Processes}

The classical pathway includes ligand activation and direct binding to estrogen responsive elements (EREs) in target gene promoters, leading to transcriptional changes in estrogen-responsive genes. The binding of the ligand to the receptor can trigger the recruitment of a variety of coregulators which can alter chromatin structure and facilitate the recruitment of the RNA polymerase II transcriptional machinery $[44,45,57,58]$.

However, ERs can also regulate gene expression without directly binding to DNA. This occurs through proteinprotein interactions with other DNA-binding transcription factors in the nucleus. In addition, membrane-associated ERs mediate nongenomic actions of estrogens, which can lead to altered functions of proteins in the cytoplasm and also to regulation of gene expression $[44,45,57,58]$. The ligand-independent pathway includes activation through other signaling pathways, such as growth factor signaling. In that case, growth factors activate protein-kinase cascades, which lead to phosphorylation and activation of nuclear ERs which, in turn, causes them to dimerize, bind DNA, and regulate genes $[44,45,57]$.

A nongenomic mechanism is characterized by rapid effects. In such type of mechanims a receptor is activated by a ligand, possibly associated with the membrane, and the complex formed is associated with the activation of various protein-kinase cascades, leading to indirect changes in gene expression which can alter functions of proteins in the cytoplasm, due to phosphorylation of transcription factors. This rapid effect can cause mobilization of intracellular calcium, generation of CAMP, production of nitric oxide, production and activation of the mitogen-activated protein kinase (MAPK) signaling pathway, activation of the phosphoinositol-3-kinase ( $\mathrm{PI} 3 \mathrm{~K})$ signaling pathway, and activation of membrane tyrosine kinase receptors $[44,45,57-59]$.

Estrogen also interacts with GPER30, a third ER discovered more recently, and the stimulation of GPER30 activates heterotrimeric $\mathrm{G}$ proteins, which, in turn, can activate multiple effectors, resulting in the production of CAMP and the activation of the tyrosine protein kinase Src, and sphingosine kinase. Src, in turn, stimulates matrix metalloproteinases (MMPs) which leads to the release of heparin-binding epidermal growth-factor-like growth factor (HB-EGF) that can transactivate EGF receptors (EGFRs). The activation of MAP kinases and PI3K, results in the activation of EGFR and leads to the expression of transcription factors $[47,60]$. When classic ERs are absent, or blocked, the effects mediated by GPER30 are maintained $[2,61]$.

Moreover, new estrogenic molecules were identified in S. haematobium total antigen as well as in the serum of infected individuals with this parasitic disease that seem to be produced by this parasite [48]. ER transcriptional activity was suppressed in urothelial cells, and ER expression was also suppressed in the bladders of mice in response to S. haematobium [49].

Schistosome eggs produce a metabolite derived from estrogen called catechol-estrogen. Cytochrome P450 oxygenases metabolize these estrogenic molecules to active quinones, that include the formation of the catechol estrogen-3,4-quinone, the major mutagenic derivative of estrogen, that causes alterations in DNA $[5,50,51]$. This ability to cause alterations in DNA is a strong inducer of carcinogenesis; in addition we [52] and others [53] demonstrated that these molecules are associated with schistosomiasis-associated bladder cancer as well as thyroid and breast cancer.

In further recent studies, the presence of estrogen-like metabolites during FGS was also associated with self-reported infertility [5]. These metabolites downregulate ER $\alpha$ and ER $\beta$ in estrogenresponsive cells in vitro because they can react with DNA to form depurinating adducts, which may generate DNA apurinic sites that, in turn, initiate mutations [49]. Our group demonstrated that the eggs of $S$. haematobium secrete these novel catechol estrogens, and that estrogens are metabolized to active quinones that modify DNA. Therefore, it is believed that the presence of parasite-derived catechol-estrogens may influence infertility during infection with Schistosoma through the blockage of estrogen receptors acting as endocrine disruptors [5,50,51]. More recently, in order to better understand this hormonal imbalance, our group investigated a serum panel of steroid hormones in Schistosoma mansoni-infected female and male hamsters [35]. Surprisingly, we found a decrease in the serum levels of E2 in infected females but not in infected

\section{Outstanding Questions}

How extensively do the effects of schistosomiasis cause infertility in their hosts?

What are the complete mechanisms by which infertility is a sequela of schistosomiasis? Is this mechanism different between S. haematobium and S. mansoni infections? How does this differ across male and female hosts? Are estrogen metabolites important for infertility-associated schistosomiasis?

Can knowledge of cancer-associated schistosomiasis, particularly estrogen metabolites, provide tools for disease control? 
males (as expected from previous results in men), in comparison with controls. These new results shed light on our understanding of the hormonal mechanism involved in schistosomiasis-associated infertility: E2 is augmented in males, explaining hypogonadism and consequent infertility, but in females E2 is below normal levels amplifying infertility observed in infected females.

\section{Concluding Remarks}

In spite of substantial evidence that points towards a key role of schistosomiasis in infertility of infected hosts, current knowledge of the fundamental mechanisms by which this phenomenon happens is scarce and fragmented. Nonetheless, the relentless progress in investigating ERs and estrogen metabolism, and their role in bioprocesses (Box 2), provides us with unprecedented opportunities to thoroughly characterize the functions of such ERs in schistosomiasis. At the core lie questions surrounding modes of action of schistosomiasis (haematobium and mansoni) causing infertility in their hosts, the complete mechanisms by which infertility is a sequela of schistosomiasis, how this sequela affects female and male hosts, the functions that schistosome estrogen catechols play in the host endocrine system, and the effects that disrupting estrogen metabolites may exert on the host, causing cancer and infertility (see Outstanding Questions). In turn, the new knowledge can be expected to provide us with a plethora of opportunities to exploit parasitehost associations to our advantage, for example, by applying cutting-edge novel ER modulators as innovative intervention strategies against schistosomes and the diseases that they cause. Two different complementary pathways probably contribute to estrogen imbalance leading to (i) infertility, and (ii) initiation and promotion of cancer progression. Future studies are necessary to study ERs in the genital organs of animals infected with Schistosoma. This should be done to determine, compared with $S$. haematobium, if $S$. mansoni also includes estrogen-related metabolites that can downregulate $E R \alpha$ and $E R \beta$, as well as the more recent estrogen receptor GPER30. Including informative estrogen-responsive cells should allow us to understand the impact of schistosomiasis on fertility.

\section{References}

1. Lindsay, T.J. and Vitrikas, K.R. (2015) Evaluation and treatment of infertility. Am. Fam. Physician 91, 308-314

2. Botelho, M.C. et al. (2015) The role of estrogens and estrogen receptor signaling pathways in cancer and infertility: The case of schistosomes. Trends Parasitol. 31, 246-250

3. Owusu-Bempah, A. et al. (2013) Genital schistosomiasis leading to ectopic pregnancy and subfertility: a case for parasitic evaluation of gynaecologic patients in schistosomiasis endemic areas. Case Rep. Obstet. Gynecol. 2013, 1-3

4. Landers, D.V. and Sweet, R.L. (1983) Tubo-ovarian abscess: Contemporary approach to management. Rev. Infect. Dis. 5, 176

5. Santos, J. et al. (2014) Urinary estrogen metabolites and self-reported infertility in women infected with Schistosoma haematobium. PLoS ONE 9, e96774

6. Bullough, C.H. (1976) Infertility and bilharziasis of the female genital tract. Br. J. Obstet. Gynaecol. 83, 819-822

7. Dzeing-Ella, A. et al. (2009) Cervical schistosomiasis as a risk factor of cervical uterine dysplasia in a traveler. Am. J. Trop. Med. Hyg. 81, 549-550

8. Botelho, M.C. et al. (2011) Targeting molecular signaling pathways of Schistosoma haemotobium infection in bladder cancer. Virulence 2, 267-279

9. Girgis, S.M. and Wassef, N.F. (1980) Bilharziasis and azoospermia. Arch. Androl. 5, 369-372
10. Figueiredo, J.C. et al. (2015) Prostate adenocarcinoma associated with prostatic infection due to Schistosoma haematobium. Case report and systematic review. Parasitol. Res. 114, 351-358

11. Bailey, S. et al. (2011) Fluke infertility: the late cost of a quick swim. J. Travel. Med. 18, 61-62

12. Kjetland, E.F. et al. (2010) The first community-based report on the effect of genital Schistosoma haematobium infection on female fertility. Fertil. Steril. 94, 1551-1553

13. Miller-Fellows, S.C. et al. (2017) Cross-sectiona interview study of fertility, pregnancy, and urogenital schistosomiasis in coastal Kenya: Documented treatment in childhood is associated with reduced odds of subfertility among adult women. PLoS Negl. Trop. Dis. 11, e0006101

14. Hotez, P. and Whitham, M. (2014) Helminth infections. Obstet. Gynecol. 123, 155-160

15. Hotez, P.J. et al. (2009) Africa's 32 cents solution for HIV/AIDS. PLoS Negl. Trop. Dis. 3, e430

16. Richardson, M.L. et al. (2014) A new mouse model for female genital schistosomiasis. PLoS Negl. Trop. Dis. 8, e2825

17. Woodall, P.A. and Kramer, M.R. (2018) Schistosomiasis and infertility in East Africa. Am. J. Trop. Med. Hyg. 98, 1137-1144

18. Talaat, M. et al. (2004) The social context of reproductive health in an Egyptian hamlet: a pilot study to identify female genital schistosomiasis. Social Sci. Med. 58, 515-524 
19. El-Mahgoub, S. (1982) Pelvic schistosomiasis and infertility. Int. J. Gynecol. Obstet. 20, 201-206

20. Abdel-Naser, M.B. et al. (2018) Schistosomiasis (bilharziasis) and male infertility. Andrologia 51, e13165

21. Colley, D.G. et al. (2014) Human schistosomiasis. Lancet 28, 2253-2264

22. Bullough, C.H.W. (1976) Infertility and bilharziasis of the female genital tract. Br. J. Obstet. Gynaecol. 83, 819-822

23. Hove, M.M. and Javangwe, T.V. (2014) Female genital schistosomiasis: pathological features and density infestation. Cent. Afr. J. Med. 60, 13-16

24. Swai, B. et al. (2006) Female genital schistosomiasis as an evidence of a neglected cause for reproductive ill-health: a retrospective histopathological study from Tanzania. BMC Infect. Dis. 6, 134

25. Khalaf, Y. (2003) ABC of subfertility. Tubal subfertility BMJ. 13, 610-613

26. Botelho, M.C. et al. (2016) Schistosoma mansoni infection impairs reproduction in mice. Proceedings of the German Society of Tropical Medicine and International Health, 7-8 October, 2016 (Germany: Bonn University)

27. Helling-Giese, G et al. (1996) Schistosomiasis in women: manifestations in the upper reproductive tract. Acta Tropica 62, 225-238

28. Poggensee, G. et al. (1999) Schistosomiasis of the female genital tract: public health aspects. Parasitology Today (Personal edn) 15, 378-381

29. Le Govic, Y. et al. (2019) Schistosoma haematobium-Schistosoma mansoni hybrid parasite in migrant boy, France, 2017. Emerg Infect. Dis. 25, 365-367

30. Owusu-Bempah, A. et al. (2013) Genital schistosomiasis leading to ectopic pregnancy and subfertility: a case for parasitic evaluation of gynaecologic patients in schistosomiasis endemic areas. Case Rep. Obstet. Gynecol. 2013, 634264

31. Balasch, J. et al. (1995) Schistosomiasis: an unusual cause of tubal infertility. Hum. Rep. 10, 1725-1727

32. Badiale, G.B. et al. (2005) Endocervical schistosomiasis. Rev. Soc. Bras. Med. Trop. 38, 351-352

33. Billy-Brissac, R. et al. (1994) Genital Schistosoma mansoni bilharziasis in women: apropos of 2 cases in Guadeloupe. Med. Trop. (Mars) 54, 345-348

34. Habib, F.A. et al. (2010) Immunohistochemical expression of oestrogen and progesterone receptors during experimental acute and chronic murine Schistosomiasis mansoni. Rev. Soc. Bras. Med. Trop. 43, 531-535

35. Oliveira, K.C. et al. (2019) Imbalance of steroid hormones in hamsters infected with Schistosoma mansoni. Endocr. Metab. Immun Disord. Drug Targets. Published online May 29, 2019. https://doi.org/10.2174/ 1871530319666190529121204

36. King, C.H. (2018) Mapping out the under-recognized burden of human infertility linked to Schistosoma haematobium infection. Am. J. Trop. Med. Hyg. 98, 937-938

37. Mbabazi, P.S. et al. (2011) Examining the relationship between urogenital schistosomiasis and HIV infection. PLoS Negl. Trop. Dis. 5, e1396

38. Kini, S et al. (2009) Schistosomiasis-induced male infertility. BMJ. Case Rep. Published online August 20, 2009. https://doi.org/10.1136/bcr.01.2009.1481

39. Abdel-Naser, M.B. et al. (2018) Schistosomiasis (bilharziasis) ova: An incidental finding in testicular tissue of an obstructive azoospermic man. Andrologia 50, e13131

40. Adisa, J. et al. (2013) Primary infertility associated with Schistosoma mansoni: a case report from the Jos plateau, north central Nigeria. African Health Sci $12,563-565$

41. Saad, A.H. et al. (1999) Possible role of Schistosoma mansoni infection in male hypogonadism. J. Egypt. Soc. Parasitol. 29, 307-323

42. Botelho, M.C. et al. (2009) Schistosoma haematobium and Schistosomiasis mansoni: production of an estradiol-related compound detected by ELISA. Exp. Parasitol. 122, 250-253

43. Lansoud-Soukate, J. et al. (1991) Role of Schistosoma mansoni bilharziasis in male hypogonadism. Pathol. Biol. (Paris) 39, 681-685

44. Heldring, N. et al. (2007) Estrogen receptors: how do they signal and what are their targets. Physiol. Rev. 87, 905-931

45. Cui, J. et al. (2013) Estrogen synthesis and signaling pathways during aging: from periphery to brain. Trends Mol. Med. 19, 197-209

46. Paterni, l. et al. (2014) Estrogen receptors alpha (ER $\alpha$ ) and beta (ER $\boldsymbol{\beta})$ : subtype-selective ligands and clinical potential. Steroids 90, 13-29

47. Prossnitz, E.R. et al. (2008) Estrogen signaling through the transmembrane $\mathrm{G}$ protein-coupled receptor GPR30. Annu. Rev Physiol. 70, 165-190

48. Botelho, M.C. et al. (2010) Schistosoma haematobium: identification of new estrogenic molecules with estradiol antagonistic activity and ability to inactivate estrogen receptor in mammalian cells. Exp. Parasitol. 126, 526-535

49. Botelho, M.C. et al. (2012) Inactivation of estrogen receptor by Schistosoma haematobium total antigen in bladder urothelial cells. Oncol. Rep. 27, 356-362

50. Botelho, M.C. et al. (2017) Estrogen catechols detection as biomarkers in schistosomiasis induced cancer and infertility. Lett. Drug. Des. Discov. 14, 135-138

51. Cardoso, R. et al. (2017) Molecular sciences estrogen metabolism-associated CYP2D6 and IL6-174G/C polymorphisms in Schistosoma haematobium infection. Int. J. Mol. Sci. 18, 2560

52. Botelho, M.C. et al. (2013) Tumour-like phenotypes in urothelial cells after exposure to antigens from eggs of Schistosoma haematobium: an oestrogen-DNA adducts mediated pathway? Int. J. Parasitol. 43, $17-26$

53. Cavalieri, E.L. and Rogan, E.G. (2011) Unbalanced metabolism of endogenous estrogens in the etiology and prevention of human cancer. J. Steroid Biochem. Mol. Biol. 125, 169-180

54. Schanz, A. et al. (2010) Genital schistosomiasis as a cause of female sterility and acute abdomen. Fertil. Steril. 93, 2075, e7-2075.e9.

55. Botelho, M.C. et al. (2017) Halting Schistosoma haematobium - associated bladder cancer. Int. J. Cancer Manag. 10, e9430

56. Botelho, M.C. (2016) Schistosoma haematobium in Guinea-Bissau: unacknowledged morbidity due to a particularly neglected parasite in a particularly neglected country. Parasitol. Res. 115, 1567-1572

57. Björnström, L. and Sjöberg, M. (2005) Mechanisms of estrogen receptor signaling: convergence of genomic and nongenomic actions on target genes. Mol. Endocrinol. 19, 833-842 
58. Vrtačnik, P. et al. (2014) The many faces

of estrogen signaling. Biochemica Medica 24, 329-371

59. Kovats, S. (2015) Estrogen receptors regulate innate immune cells and signaling pathways. Cell Immunol 294, 63-69

60. Cato, A.C.B. et al. (2002) Rapid actions of steroid receptors in cellular signaling pathways. Science's STKE 2002, re9.

61. Prossnitz, E.R. and Maggiolini, M. (2009) Mechanisms of estrogen signaling and gene expression via GPR30. Mol. Cell Endocrinol. 308, 32-38 